\title{
Dissecting learning
}

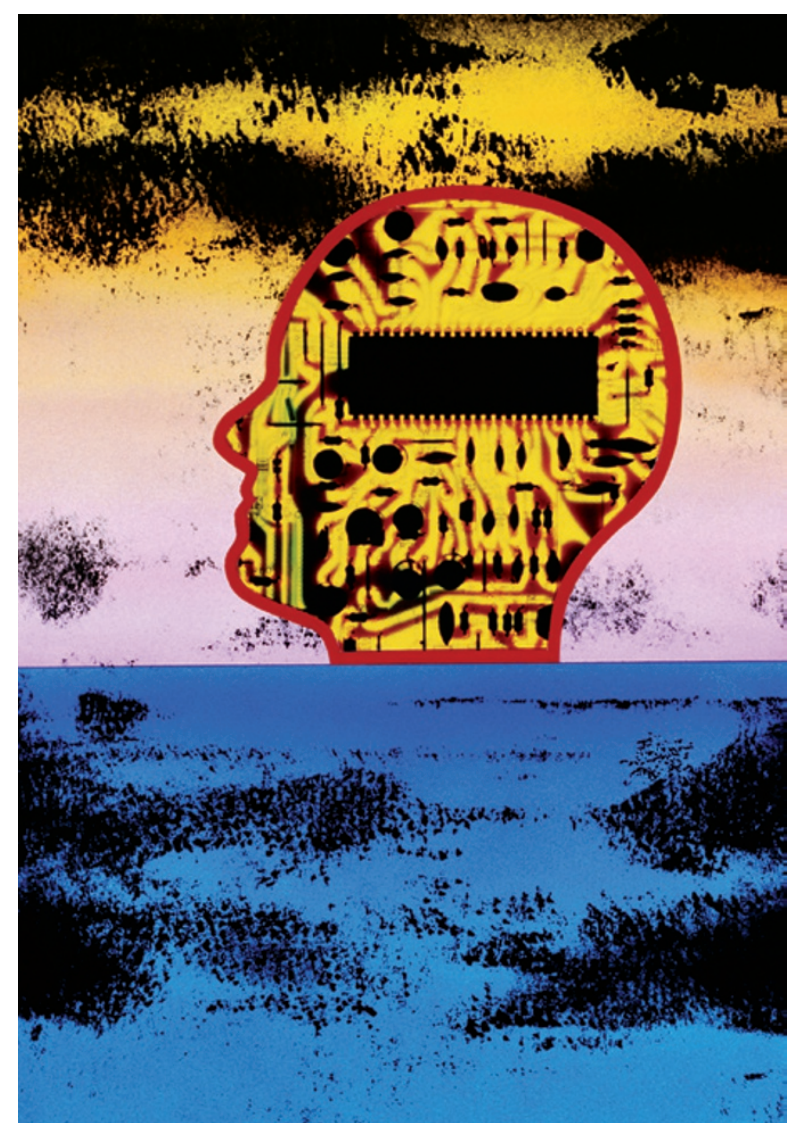

Patients and animals with spatially restricted lesions have enabled researchers to study the contribution of individual brain regions to cognitive functions. There is great hope that the development of methods to more selectively disrupt individual neuronal circuits will resolve many outstanding issues in the field. Two new papers demonstrate how such advances can provide new insights into the functions of individual cortical circuits.
Place cells in the hippocampal CA1 area encode an animal's spatial position in its environment. The continual modification of CA1 'place fields' is determined by information from the entorhinal cortex that arrives either directly or through an indirect multisynaptic route that involves the CA3. Moser and colleagues' previous work indicated that the direct pathway could by itself support place-field formation; here they asked whether this pathway is necessary for place-field tuning. They used a neurotoxin that specifically targets the part of the rat entorhinal cortex that projects directly to CA1 to create a lesion in this area. Electrophysiological recordings from CA1 place cells showed that the lesion reduced spatial tuning in CA1 during exploration of familiar or novel environments. Recordings from CA3 cells projecting to CA1 were normal, confirming that the effects of the lesion were due to the loss of the direct input and, thus, that this pathway is required for the tuning of spatial representations in CA1.

Although Moser and colleagues took great care to ensure that these lesions were selective, it would be of great benefit to find a method that would allow individual pathways to be targeted with even more precision. The second paper, by Tonegawa and colleagues, describes the development of such a technique. The authors generated triple-transgenic mice in which the expression of a toxin that abolishes synaptic transmission was placed under the control of two promoters that targeted it specifically to the synapses of the indirect pathway and a promoter that could be repressed by doxycycline (Dox) treatment. Four weeks after Dox removal, synaptic transmission in the indirect pathway was significantly impaired, allowing the authors to examine the importance of the indirect pathway in various memory paradigms.

The authors observed no impairments in the acquisition or recall of spatial learning in a slowly-aquired task, suggesting that the direct pathway can support this function on its own. However, there were significant impairments in the formation of rapid one-trial contextual fear memory and in pattern-completion-based recall. Furthermore, the authors observed a decrease in spatial tuning in CA1 place cells in both novel and familiar environments, suggesting that the indirect pathway is also required for the rapid contextual formation of representations.

Although these studies do not give us definitive answers about the roles of these direct and indirect pathways in spatial memory, they do bring us closer to understanding these complicated circuits. In addition, the use of ingenious genetic methods will help to clarify the sometimes conflicting results of lesions and pharmacological experiments.

Katherine Whalley

ORIGINAL RESEARCH PAPERS Brun, V. H. et al.

Impaired spatial representation in CA1 after lesion of direct input from entorhinal cortex. Neuron 57, 290-202 (2008) | Nakashiba, T. et al. Transgenic inhibition of synaptic transmission reveals role of CA3 output in hippocampal learning. Science 27 Jan 2008 (doi:10.1126/ science.1151120) 